\title{
Las ocupaciones en los servicios de limpieza y de estética: algunas pistas para reflexionar en torno de la movilidad laboral de las mujeres de sectores populares en Argentina
}

\author{
Débora Gorban \\ ICI-UNGS / CONICET \\ dgorban@ungs.edu.ar \\ Ania Tizziani \\ ICI-UNGS / CONICET \\ atizzian@ungs.edu.ar
}

Resumen: Este artículo se propone indagar en torno de los patrones de segregación multidimensionales de género y sector socioeconómico en el mundo del trabajo para el caso de la ciudad y área metropolitana de Buenos Aires. Para ello analizaremos las experiencias de trabajo de las mujeres de sectores populares centrándonos en los servicios de limpieza y en las ocupaciones de estética. Se trata de las ocupaciones más representativas y también unas de las más desfavorable del mercado de trabajo (en términos de niveles de salario y condiciones de trabajo). En base a una investigación que venimos desarrollando desde el 2009 buscamos no solo caracterizar estas ocupaciones sino la manera en que las trabajadoras se desplazan entre ellas y los significados que le atribuyen a esta movilidad.

Palabras clave: Movilidad, género, segregación, limpieza, estética, Argentina. 
Cleaning and aesthetics occupations: Inquiries about women from popular sectors'labor mobility in Argentina

Abstract: This article aims to investigate the multidimensional gender and class segregation patterns in the world of work for the case of the city and metropolitan area of Buenos Aires. In that sense, we will analyse the work experiences of women from popular sectors, focusing on cleaning services and aesthetic occupations. These are the most representative occupations and also one of the most unfavourable in the labour market (in terms of salary and working conditions). Based on an research program that we have been developing since 2009, we seek not only to characterize these occupations, but also the way in which workers move among them and the meanings attributed to this mobility.

Keywords: Mobility, gender, segregation, cleaning, aesthetics, Argentina. 


\section{Introducción y planteo del problema}

En la Argentina, como en la mayoría de los países latinoamericanos, el trabajo remunerado de cuidados, vinculado fundamentalmente a los servicios, es desarrollado principalmente por las mujeres de los grupos sociales más desfavorecidos. Tal como lo recuerda Arango (2011), estas actividades han constituido una de las principales críticas del feminismo al concepto de trabajo, en un esfuerzo por pensar herramientas conceptuales que permitan comprender las particularidades del trabajo que realizan las mujeres. En ese sentido, el concepto de cuidados abarca el conjunto de actividades destinadas a garantizar el sostenimiento cotidiano de la vida humana, tanto en su aspecto físico como emocional (Tronto, 2015; Arango, 2011; Molinier, 2005). Este tipo de tareas se realizan, en su gran mayoría, en el espacio doméstico y han sido identificadas como "tareas de mujeres" y esencializadas como tales justificando desde allí, entre otros argumentos, su carácter no remunerado. Fuera del ámbito familiar, se trata de actividades remuneradas, ya sea que se desarrollen en otros espacios domésticos (trabajo doméstico remunerado) o en empresas, instituciones, entre otros lugares.

Tal como sostiene Arango (2011), entre otras autoras, la conceptualización de estas actividades como trabajo de cuidados ha permitido cuestionar su asociación como "actividades de mujeres", permitiendo también dar cuenta de la manera en que se inscriben tanto en un orden binario de género, como dentro de jerarquías de clase, raza y etnicidad. Estas actividades son frecuentemente realizadas por mujeres que ocupan posiciones subalternas, constituyendo una de las principales fuentes de empleo femenino urbano, para este grupo. En Argentina, en su mayoría, se trata de ocupaciones que ofrecen las condiciones más desventajosas del mercado de trabajo, con bajos niveles de salario, altos niveles de informalidad y precariedad y escaso reconocimiento social. Desde nuestra perspectiva, el análisis de la manera en que se organizan y se estructuran estas ocupaciones, constituye una estrategia privilegiada para el estudio de los fuertes condicionamientos de género y clase que enfrentan las mujeres más desfavorecidas en su inserción laboral. En base a una investigación cualitativa que desarrollamos actualmente en el Área Metropolitana de Buenos Aires, nos proponemos en este artículo caracterizar las experiencias laborales de las mujeres que se insertan en algunas ocupaciones que forman parte del universo de los cuidados: los servicios de limpieza (doméstica y no doméstica) y los servicios de estética. Prestaremos particular atención a cómo se construyen los desplazamientos de las trabajadoras a través de estas diversas actividades y cuál es el significado que le atribuyen a esa movilidad.

Esta investigación forma parte de un proyecto colectivo más amplio, cuyo objetivo es indagar sobre la manera en que las mujeres de sectores populares se 
posicionan en y frente al mundo del trabajo ${ }^{1}$. Nace como continuación de investigaciones anteriores, que desarrollamos desde el año 2009, centradas en el análisis de las trayectorias y condiciones laborales de las mujeres que se insertan en el trabajo doméstico remunerado y el tipo de relaciones que establecen con sus empleadores ${ }^{2}$. Como en otros análisis en ese campo de estudios, en esas investigaciones, el servicio doméstico es pensado como un lugar crítico para reflexionar en torno de la manera en que se construyen y se reproducen, en la práctica cotidiana, mecanismos de diferenciación y de jerarquización social, basados en el género, la clase, la etnicidad y la racialidad (Rollins, 1985, Romero, 2002, Hondagneu Sotelo, 2007 y Chaney \& Castro, 1993, Brites, 2001 para el caso de América Latina, entre otros). Permite así el análisis de segregaciones multidimensionales en el mundo del trabajo (Jaunait \& Chauvin, 2012), que restringen y condicionan fuertemente el horizonte de inserciones posibles para las mujeres de menores recursos. Nuestro objetivo es, en este artículo, seguir profundizando esa línea de indagación.

En las secciones siguientes nos referiremos, en primer lugar, a la metodología empleada en el estudio que es la base del presente artículo. Luego realizaremos un recorrido por la literatura y la manera en que ha sido abordado el análisis de las inserciones laborales de las mujeres en América Latina y en Argentina en particular, lugar en donde se desarrolla nuestro trabajo de campo. Posteriormente, expondremos algunos resultados de nuestro estudio en relación a las formas que adquieren las movilidades de las mujeres que se insertan en las actividades laborales consignadas, los servicios de limpieza y de cuidados personales, y la manera en que las perciben. Por último, plantearemos algunas reflexiones finales.

\section{Metodología}

Las reflexiones que siguen se basan en una investigación iniciada en 2009, de tipo cualitativo, que se desarrolló en varias etapas. Entre los años 2009 y 2011 , realizamos en la ciudad de Buenos Aires una serie de 25 entrevistas en profundidad con trabajadoras domésticas. Esas trabajadoras fueron contactadas, por un lado, en diferentes organizaciones que intervienen en este sector (asociaciones, sindicatos), donde además conversamos con las autoridades y coordinadores de

1 Se trata del proyecto PICT "Las decisiones, estrategias y experiencias laborales de las mujeres de sectores populares. Un estudio exploratorio en el AMBA", MINCyT/UNGS (2017-2020).

2 Estos proyectos son "Procesos de construcción y legitimación de las jerarquías sociales en la Argentina actual: empleadas y empleadoras del servicio doméstico" (Conicet-UNGS), "Los rostros de María. Representaciones e imágenes del servicio doméstico en la Argentina del siglo xx., PIP 11220110100056, CONICET. 2012- 2014. Proyecto "Entre el afecto y el trabajo. Un estudio sobre las experiencias y condiciones de trabajo de las empleadas domésticas en la Ciudad de Buenos Aires", PICT 07-00258, ANCPYT. 2009- 2011. 
dichos espacios y realizamos observaciones de las actividades que se desarrollan en sus locales. Por otro lado, para diversificar el perfil de las mujeres entrevistadas, durante cuatro meses realizamos observaciones y una serie de entrevistas informales, en su mayoría repetidas, en dos plazas de la ciudad, donde pudimos contactar empleadas domésticas que realizan (en general no exclusivamente) tareas de cuidado de niños. Las edades de las trabajadoras contactadas variaban entre 16 y 65 años al momento de realizar las entrevistas.

Los datos obtenidos durante ese trabajo de campo se complementan con un estudio más reciente, iniciado en el año 2016, sobre las experiencias y trayectorias laborales de las mujeres que se insertan en diversos servicios de limpieza no doméstica y de cuidados personales. En relación con la limpieza no doméstica, realizamos, hasta la fecha, una serie de diez entrevistas en profundidad con trabajadoras que se insertan como mucamas de hotel o como empleadas de maestranza en empresas, fábricas y diversas instituciones. Se trata de mujeres de entre 39 y 56 años de edad, que, en su mayoría, residen y trabajan en el conurbano bonaerense. Paralelamente, en relación con los servicios de peluquería y estética se realizaron, hasta el momento, una serie de ocho entrevistas en profundidad a mujeres que se dedican a estas actividades, en salones y en atención a domicilio. Algunas de ellas se encuentran actualmente ejerciendo tareas de docencia en una escuela de formación en peluquería y manicuría, en donde también realizamos observaciones participantes. Las mujeres entrevistadas para este caso tienen entre 24 y 50 años. Este trabajo se complementa con observación en los salones de peluquería y en los domicilios de las trabajadoras. En el marco de ese conjunto de entrevistas, indagamos en torno a diferentes ejes, que intentamos articular en este texto: las experiencias y condiciones laborales, pasadas y presentes, de las mujeres contactadas, sus trayectorias familiares, residenciales y educativas. Para el presente artículo hemos partido del análisis realizado en función de los datos de nuestra investigación sobre servicio doméstico para desde allí analizar las entrevistas que conforman la etapa del trabajo de campo en curso sobre los servicios de limpieza no doméstica y cuidados personales.

\section{Algunas problemáticas en torno de la inserción laboral de las mujeres de sectores populares}

Diferentes estudios han destacado la persistencia de una marcada segregación de género y clase social en el mercado de trabajo argentino de las últimas décadas (Cortés, 2012). Por un lado, pese al mejoramiento de los indicadores laborales que se registró en el país hasta fines de la década pasada, las desigualdades de 
género siguen siendo importantes (Cortés, 2012; Novick et al, 2008; Bertranou y Casanova, 2013). Como en otros países de la región, las mujeres están más afectadas que los trabajadores varones por la desocupación, la subocupación, la precariedad e informalidad laboral (CEPAL, 2014). De la misma manera, diferentes estudios constatan la delimitación de territorios masculinos y territorios femeninos en la estructura ocupacional, la persistencia de elevadas brechas salariales, incluso en trabajadoras/es de iguales niveles de calificación y cargo jerárquico, así como la menor presencia de las mujeres en cargos de supervisión, gerenciales y de dirección (Novick et al, 2008).

Uno de los elementos centrales en la configuración de estos patrones de desigualdad de género en el mercado de trabajo, destacado por una amplia literatura, es la profunda imbricación entre el trabajo reproductivo y productivo en los itinerarios femeninos y la articulación de las decisiones laborales y las dinámicas familiares (Wainerman, 2002; Barrancos \& Goren, 2002, entre otros). El peso de las responsabilidades socialmente asignadas a las mujeres en las tareas reproductivas condiciona tanto las posibilidades de participación en actividades remuneradas fuera del hogar, como el tipo inserción a la que pueden acceder. Al mismo tiempo, la permanencia de estereotipos de género que definen roles, competencias y saberes diferenciados para varones y mujeres (con valorizaciones desiguales), contribuye también a restringir el universo de inserciones laborales accesibles a las mujeres (Novick et al, 2008).

Así, en la Argentina, las mujeres urbanas se concentran principalmente en el comercio y los servicios, y allí, en actividades consideradas como "típicamente femeninas", es decir, aquellas que son definidas como extensión de las tareas reproductivas socialmente atribuidas a las mujeres. Para las mujeres de sectores medios, la enseñanza y los servicios sociales y de salud son los sectores más representativos. En el caso de aquellas pertenecientes a los sectores populares, con menores niveles de educación formal, los servicios domésticos y de cuidados constituyen las inserciones más frecuentes (Dávalos, 2013; Contartese y Maceira, 2005).

Estas inserciones diferenciadas de las mujeres según el sector socio-económico sugieren la necesidad de articular el análisis de estos patrones de segregación de género con otras dinámicas de configuración de las desigualdades, en particular aquellas vinculadas a la pertenencia de clase. En efecto, son las mujeres de bajos ingresos las que enfrentan los horizontes de inserción laboral más acotados y con condiciones más desventajosas. Esto se relaciona principalmente con los escasos recursos que pueden movilizar para hacer frente a las responsabilidades reproductivas socialmente atribuidas. El impacto de estas responsabilidades en 
las trayectorias laborales femeninas depende sin duda de arreglos y negociaciones al interior de los hogares, pero también del acceso (diferencial) a servicios fuera del hogar, sean comunitarios o de mercado: trabajo doméstico remunerado, guarderías e instituciones de cuidado de enfermos y ancianos, el acceso a tecnología doméstica, etc. (Jelin, 2010). El hecho de que la oferta de servicios de este tipo esté centrada en los mecanismos de mercado, contribuye a reproducir estos patrones de segregación por género y sector socio-económico en el mercado de trabajo.

En un estudio reciente, focalizado en el Área Metropolitana de Buenos Aires, Francisca Pereyra y Ariela Micha analizan en detalle la situación ocupacional de las mujeres de sectores populares en las edades activas (de 15 a 60 años) $)^{3}$. Estas autoras muestran, entre otros rasgos, que las mujeres de estos estratos registran tasas de actividad menos elevadas que sus pares de sectores medios y altos, se ven más afectadas por la desocupación, la subocupación demandante y se insertan en actividades atravesadas por altos niveles de informalidad. En el caso de las ocupadas, perciben, además, salarios considerablemente más bajos que las trabajadoras de otros estratos sociales: los ingresos promedio de la ocupación principal de estas mujeres representan un tercio de lo que perciben sus pares de nivel socio-económico alto. Se concentran, en el año 2016, principalmente en el servicio doméstico (cerca de 22\%), en la venta y comercialización (16\%) y en los servicios de limpieza no domésticos (10\%) y en los servicios de estética (6,5\%) (Micha y Pereyra, 2017).

En las trayectorias y los relatos de las mujeres que entrevistamos en nuestra investigación sobre el servicio doméstico, dos elementos aparecían muy frecuentemente como condicionando las posibilidades de inserción laboral. En primer lugar, ingresos muy tempranos en el mercado de trabajo, vinculados a situaciones de vulnerabilidad en las familias de origen, que implicaban la interrupción de la escolarización formal. En segundo lugar, las dificultades para compatibilizar el trabajo reproductivo y productivo. Esas dificultades las conducen hacia una participación intermitente en el mercado de trabajo y hacia ocupaciones que pueden ofrecer cierta flexibilidad, con altos niveles de precariedad e informalidad, como la limpieza doméstica "por horas". Estos dos elementos (entre otros) van configurando trayectorias inestables, que se desarrollan en actividades de baja calidad, consideradas como no calificadas (ni calificantes) (Gorbán y Tizziani, 2014). Sin embargo, el servicio doméstico no es la única actividad por la que las mujeres que entrevistamos transitaron. En este artículo nos centraremos en dos

3 En ese estudio, la clasificación por nivel socio-económico se basó en las características de los hogares, tomando como criterio principal el máximo nivel educativo alcanzado por el principal sostén del hogar (Micha y Pereyra, 2017). 
de las ocupaciones que aparecen más frecuentemente en sus trayectorias y en sus relatos: servicios de limpieza no doméstica y servicios de estética, en particular, las actividades de peluquería.

En relación con estas ocupaciones, buscamos en primer lugar caracterizar las experiencias de trabajo de las mujeres que se insertan en ellas. En segundo lugar, nos interesará explorar las posibilidades de acceso a diferentes instancias de capacitación y formación profesional, como uno de los elementos centrales que configuran los desplazamientos través de estas ocupaciones. Esta última dimensión está fuertemente asociada al reconocimiento que las trabajadoras encuentran en el ejercicio estas diferentes actividades y tiene, desde nuestra perspectiva, un impacto significativo en la manera en que evalúan y perciben la movilidad entre ellas.

\section{Las ocupaciones de la limpieza}

En su definición estadística, el sector de servicios de limpieza abarca un conjunto de categorías ocupacionales muy heterogéneas: trabajadoras/es domésticas/os, mucamas/os de hotel, encargadas/os de edificios, auxiliares de limpieza en empresas, fábricas o locales, barrenderas/os, etc ${ }^{4}$. En los relatos de las trabajadoras, estas diferentes actividades se presentan como fronterizas, en un continuo de ocupaciones que articula los espacios domésticos y los no domésticos. La mayoría de ellas transitó por empleos de limpieza o cuidados dentro y fuera de los hogares particulares: fueron empleadas domésticas, fueron contratadas como auxiliares en geriátricos, se ocuparon de la limpieza de clínicas, hoteles, pequeñas industrias o comercios. Sus relatos muestran un desplazamiento muy naturalizado a través de este continuo de ocupaciones, que muchas veces se superponen.

"Yo estoy trabajando en limpieza" comenta Silvia, una mujer de 42 años, en uno de nuestros primeros encuentros. En esa frase engloba varios empleos "por horas" en casas particulares y un trabajo a medio tiempo en la limpieza de un edificio, contratada por un consorcio. Es esa tarea común, la limpieza, la que le da un contenido específico a las actividades que ejerce y define el universo de inserciones laborales por las que ha transitado. En el relato de Silvia, como en el de muchas otras entrevistadas, esa tarea comunica los espacios domésticos y no domésticos y le da una coherencia a sus trayectorias laborales: permite restituirlas a través de una continuidad.

Es también el caso de Andrea, de 43 años, que es empleada de maestranza en una fábrica papelera en el conurbano bonaerense en el momento en que

4 Ver el Clasificador Nacional de Ocupaciones, Instituto Nacional de Estadísticas y Censos, Argentina: $<$ http://www.indec.gov.ar/ftp/cuadros/menusuperior/eph/EPHcontinua_CNO2001_reducido_09.pdf >. 
realizamos las entrevistas. Su relato da cuenta de una trayectoria laboral particularmente inestable, que se interrumpe al momento de sus dos matrimonios y los nacimientos de sus cuatro hijos, y también en períodos de desempleo, de "mucha necesidad". Siempre se desempeñó en actividades de limpieza: en casas particulares, en un shopping center, en una fábrica de acetileno, en un criadero de pollos, en el local de una "bailanta". La limpieza unifica, en su relato, todos los empleos que ejerció y le da una coherencia a su trayectoria. Ésta es una dimensión importante de la manera en que las mujeres con las que conversamos revalorizan y legitiman sus carreras laborales. En la acumulación de experiencias en torno de la misma actividad pueden reivindicar la adquisición de una serie de saberes (prácticos, técnicos y relacionales) que las vuelven idóneas para los puestos que ocupan. Esa coherencia les permite mostrar un rol activo en la construcción de sus trayectorias laborales, reivindicar un margen de autonomía y de elección entre los diferentes empleos que forman parte de ese horizonte de inserciones laborales posibles.

Sin embargo, esta naturalización no elimina las diferencias que el lugar de trabajo impone a la experiencia cotidiana de las trabajadoras. Estas diferencias no están ancladas en la naturaleza de la tarea realizada, sino en las condiciones en las que se realiza. Tal como hemos analizado en otro lugar (Tizziani, 2011; Tizziani y Gorban, 2014), cuando las tareas de limpieza se desempeñan en las casas de familia, esas condiciones se definen en una negociación individualizada con las/ os empleadoras/es, con escasas intervenciones externas; en las actividades de limpieza no doméstica, las normas que rigen los procesos de trabajo aparecen más estandarizadas. En el testimonio de Miriam, una mujer de 48 años, al comparar sus experiencias en el servicio doméstico con el trabajo de mucama en un hotel:

Cuando uno trabaja en una casa, es como más personal el trato y en ese lugar [el hotel] era como un colegio, hay muchas reglas, vos tenés que cumplir con ciertas cosas. En una casa vos podés arreglar, la persona te dice "bueno, esto yo lo quiero así", pero es una, dos cosas, después el resto es todo limpiar y que esté en condiciones. En cambio en el hotel había una regla para cada cosa, qué productos tenés que usar, los cuatro pasos para hacer la cama, todo anotado, todo como te decía la gobernanta.

Como muestra el testimonio anterior, y los discursos de muchas entrevistadas, el trabajo en el servicio doméstico responde a pautas fuertemente personalizadas. Como en el caso de Miriam, esa personalización les permite muchas veces alcanzar cierta independencia en la realización cotidiana de sus tareas, pero también constituye uno de los aspectos más problemáticos del trabajo en el sector. Los términos de los contratos entre empleadas y empleadoras/es, en lo que se 
refiere a las tareas (y los modos de llevarlas a cabo), los horarios y días de trabajo, no son fijos sino variables y se configuran en una negociación permanente entre ambas partes. Se modifican frecuentemente en función de la evolución de las dinámicas de organización de los hogares empleadores. Porque los actores que pone en contacto esa negociación se encuentran en posiciones sociales y económicas muy desiguales. La "casa particular", en tanto espacio íntimo y privado del empleador, responde a sus propias reglas y sus propias necesidades y se resiste a incorporar regulaciones públicas y colectivas cuya aplicación parece menos problemática en otras ocupaciones asalariadas. De hecho, muchas de las mujeres entrevistadas, como Gabriela cuando ingresa en una fábrica papelera, acceden a un empleo regularizado en los servicios de limpieza no doméstica, después de varias experiencias "limpiando casas".

Para aquellas que se insertan (o se insertaron) en esta ocupación -la limpieza doméstica-, las dificultades para gestionar las negociaciones muy desiguales que establecen con las y los empleadoras/es, donde se definen buena parte de las condiciones de trabajo, es una de las problemáticas centrales que enfrentan en ella. Y también el origen principal de la conflictividad que atraviesa los relatos de las mujeres entrevistadas sobre sus experiencias laborales (Tizziani, 2011). El relato de Silvia, que se refiere en el pasaje siguiente a sus primeros empleos en el sector, es un ejemplo de esas relaciones conflictivas que son características del servicio doméstico:

Ahí estuve dos años. Era insoportable. Yo tenía mi piecita. Ella [la empleadora] me decía vos tenés tus horas de descanso de tal a tal hora. Bueno, aprovechaba esas horas, me bañaba, me preparaba algo para tomar, miraba la tele... [mímica de golpes en la puerta] ay, çno venís y me ayudás? Y así, me cansé, busqué algo, calladita la boca, me busqué algo. Y me retuvo el sueldo, no me acuerdo si fueron dos o tres semanas de trabajo que no me pagó. Me dijo, después venite a buscar el sueldo, quería que yo vaya... no fui nada, guardátela, si te hace falta. Después trabajé en otra casa, con un matrimonio joven que tenía dos nenas, pero ya me querían encajar las nenas y yo dije no, no, yo vengo de empleada doméstica, de niñera no, de ahí también me fui rápido.

En cambio, en las ocupaciones que se desarrollan por fuera del espacio doméstico, el aislamiento y la dimensión personalizada de la relación laboral que caracterizan el trabajo en casas particulares son menos pronunciados. Las normas

5 El nivel de empleo no registrado que se constata en el servicio doméstico es uno de los más elevados del mercado de trabajo argentino. Sin embargo, mostró, en los últimos quince años, un cambio de tendencia significativo, pasando de cerca de 95\% en 2005 a 72\% en 2016 (Pereyra, 2017). En los servicios de limpieza no doméstica, el empleo registrado alcanza niveles significativamente más elevados y se sitúa, en el año 2016, en torno del 80\% (Fuente: Encuesta Permanente de Hogares, tercer trimestre de 2016, <www.indec.gov.ar>). 
que regulan los procesos de trabajo son explícitas y estandarizadas: "una regla para cada cosa", comenta Miriam sobre el hotel. También es la experiencia de Andrea, cuando se refiere a su trabajo de limpieza en el criadero de pollos: "Había que limpiar, desinfectar todo y en cada sector era diferente lo que había que hacer, me acuerdo que en "reproducción" era todo muy delicado, tenías que entrar con alguien que te decía paso a paso lo que había que hacer, era un quilombo".

En todos los casos, estos puestos se caracterizan por la intensidad del ritmo y la carga de trabajo, así como por el desgaste físico que requiere el desarrollo cotidiano de las tareas. Las trabajadoras se refieren frecuentemente a este esfuerzo físico cuando se trata de dar cuenta de sus proyectos de movilidad hacia otro tipo de empleos, proyectos que nacen de la necesidad de buscar "algo más liviano" como sostiene Sandra, una de nuestras entrevistadas de 51 años que combinaba, en el momento de las entrevistas, un empleo a medio tiempo en la limpieza de oficinas y dos "casas particulares". En ese sentido, muchas trabajadoras mencionan diferentes problemas de salud ligados al ejercicio de estas actividades: dolores en la cintura o en la espalda, problemas en las articulaciones, contracturas, etc. Karen Messing (2016) constata, en su estudio sobre las ocupaciones de limpieza en Francia y Canadá, los riesgos físicos que las caracterizan, asociados al peso de los carros que las trabajadoras deben transportar, las utilización de materiales mal adaptados para los espacios y las superficies que deben limpiar y el manejo de productos químicos nocivos para la salud, entre otros factores. Estos riesgos están muy presentes en los relatos de las trabajadoras, pero son en general subestimados tanto por los empleadores como por muchos estudios en torno de estas ocupaciones (Messing, 2016).

Por otro lado, la rigidez de los horarios, a veces nocturnos o a contraturno hace que estas ocupaciones sean muy difíciles de articular con las actividades de cuidado en sus propios hogares, de las que la mayoría de las trabajadoras son las principales responsables. Es la experiencia de Gabriela por ejemplo, quien en el momento de las entrevistas trabaja en un puesto de limpieza y portería de una fábrica papelera, tres días a la semana en turnos de doce horas. El cuidado de su hija de nueve años requería una serie de arreglos cotidianos muy complejos e inestables, que implicaban, según los días, la presencia de su madre, de su hijo mayor de 18 años y la ayuda ocasional de vecinas o amigas.

No todas las trabajadoras están en condiciones de responder a estas dos exigencias que caracterizan la mayoría de los empleos en los servicios de limpieza no doméstica. Así, las decisiones laborales, estos desplazamientos a través del conjunto de ocupaciones del sector, están estrechamente asociados al ciclo de vida de las trabajadoras, a sus situaciones familiares y a la organización cotidiana de sus 
hogares. Como contrapartida, se trata de puestos más estables que aquellos ejercidos en la limpieza doméstica, en los que tienen posibilidades de acceder a remuneraciones más elevadas y a los beneficios asociados a la condición de asalariadas. Se trata, además, de espacios laborales que las trabajadoras pueden capitalizar en términos de formación. En el relato de Miriam que transcribimos más arriba, el hotel es asimilado a un "colegio", una experiencia de aprendizajes múltiples, a veces formalizados en capacitaciones de varios meses: "yo siempre dije, una vez que pasás por ahí y por esa señora [la gobernanta], podés trabajar en cualquier lado".

Así, el trabajo en las ocupaciones de limpieza implica, para aquellas que lo ejercen, experiencias laborales muy diferentes según el lugar en el que se desempeñen. Sin embargo, todas estas ocupaciones comparten una misma problemática: están fuertemente afectadas por la invisibilidad y la falta de reconocimiento social. En las fábricas u oficinas, en hoteles u hospitales, las actividades de limpieza ocupan a las trabajadoras (y los trabajadores) de menor jerarquía y se efectúan por fuera del ritmo de vida propio del lugar en el que se realiza. Es una labor que se lleva a cabo en los márgenes: "antes" (muy temprano a la mañana) o "después" (en horarios nocturnos), rara vez durante el tiempo productivo de esos lugares (Brétin, 2000). Como en el caso de muchas trabajadoras domésticas, los operarios y las operarias de limpieza son considerados/as como figuras externas, discretas y silenciosas, "una imagen que revela un orden social, práctico y simbólico que subordina esta actividad a aquellas consideradas como dominantes" (Brétin, 2000: 99). Como nos cuenta Miriam, sobre su experiencia en el hotel:

"A nosotras no nos veían, teníamos contacto nada más con los chicos de mantenimiento. Unos entraban por una puerta, usaban un ascensor, nosotras otro, entonces no te cruzabas con nadie, estábamos como en un mundo paralelo [... ] Y con los pasajeros no teníamos mucho contacto, tampoco nos dejaban hablar con ellos, lo que nos pedían era no tener confianza con los señores [risas], porque a veces había señores que también llamaban... ya más o menos entendés, te llaman, te dicen ¿puede venir a la habitación? Eso lo teníamos prohibido si no pasábamos por la gobernanta”.

En el relato de Miriam, el "mundo paralelo" de la limpieza (y de las mujeres que limpian), es un mundo subordinado, separado de otros trabajadores y trabajadoras, con circulaciones y ritmos diferentes. Ese mundo de la limpieza es central en el funcionamiento del hotel, y sin embargo no puede verse ${ }^{6}$. Un mundo aparte, invisible y discreto, que es también el lugar de la feminidad: de la femi-

6 Débora Garazi (2014) analiza, para el caso de la ciudad de Mar del Plata, la manera en que diversas características del trabajo doméstico (no remunerado) se hacen extensivas al trabajo de las mucamas de hotel, contribuyendo a su invisibilidad y desvalorización. 
nidad en tanto rol social, pero también en tanto cuerpo femenino que trabaja, cuerpo femenino sexualizado que supone un riesgo que debe ser controlado.

Las actividades de limpieza se transforman cuando se trasladan del espacio doméstico a los espacios externos. En cierta medida, adquieren algunos de los rasgos a través de los cuales se representa el trabajo masculino: impersonal, regulado y estandarizado en los procesos de trabajo, con aprendizajes que pueden capitalizarse, asociado al esfuerzo físico (Willis, 1988). En términos de la experiencia y las condiciones de trabajo, como vimos, los servicios de limpieza no doméstica pueden ofrecer puestos más ventajosos: regularizados, estables y con remuneraciones más elevadas. Se trata sin embargo de puestos a los que no todas las trabajadoras pueden acceder: los horarios rígidos, de difícil compatibilización con las responsabilidades de cuidados, así como la intensidad de la carga y el ritmo de trabajo limitan los desplazamientos hacia estas ocupaciones. Pese a estas diferenciaciones, el conjunto de estas actividades de limpieza (doméstica y no doméstica) es considerado como "típicamente femenino", cristaliza uno de los roles femeninos por excelencia, que se extiende y se prolonga de los espacios domésticos hacia los no domésticos. Se trata de actividades que se desarrollan en los márgenes, marcadas por una fuerte desvalorización. Este rasgo que el conjunto de las ocupaciones de este sector tienen en común contribuye a unificar el sentido que las trabajadoras atribuyen al ejercicio de estas actividades. La movilidad entre ellas parece ser percibida como una movilidad entre ocupaciones del mismo tipo. En los relatos de la trabajadoras, se trata de una movilidad fronteriza, horizontal, más que un mejoramiento significativo del modo en que se posicionan en el mercado de trabajo.

\section{Estética, peluquería y cuidado corporalः trayectorias aspiracionales}

A lo largo de las últimas dos décadas el sector de servicios de estética y peluquería ha crecido enormemente. En Argentina, según un estudio del propio sector, existen 42 mil locales de peluquería, los cuales suman más de 120 mil puestos de trabajo y reciben un promedio de 250 millones de visitas de clientas/es por año ${ }^{7}$. Este dinamismo también se observa en la numerosa oferta de escuelas de formación profesional y técnica existentes tanto en la Ciudad de Buenos Aires como en el Conurbano Bonaerense. Recorrer las calles de la ciudad y de las localidades del conurbano, supone encontrar pequeños y grandes salones, ubicados en los más disímiles barrios, en cuanto a sus características socioeconómicas, que ofrecen

7 Información difundida por L’Oreal a raíz del lanzamiento del Programa de formación en peluquería «Belleza Por Un Futuro» en la Gacetilla L'Oreal Beauty Report N², 22 de noviembre de 2016 
sus servicios a una variedad aun mayor de clientas y clientes. Convirtiéndose de esta manera en una inserción laboral en crecimiento y que, a diferencia del servicio doméstico, no constituye una inserción con mayor representación de mujeres, sino que aquí encontramos un porcentaje significativo de varones. En efecto allí, la presencia masculina es representativa en términos del lugar jerárquico que los varones ocupan en este campo profesional, ya que aparece como un oficio que en su representación hegemónica esta identificado con carreras exitosas de varones.

Como dijimos en la introducción, esta ocupación aparece como una inserción laboral disponible en el horizonte de las mujeres de sectores populares, y tal como se desprende de nuestro campo con trabajadoras del servicio doméstico, lo hace en dos sentidos: como proyecto de formación que habilitaría un horizonte laboral futuro; o bien como alternativa laboral a otras actividades (limpieza doméstica y no doméstica).

En primer lugar, observamos que los servicios de estética y peluquería representan la posibilidad de un trabajo con ciertas características contrapuestas a los servicios de limpieza, principalmente en relación al tipo de formación que requiere, al tipo de tarea que se desarrolla - vinculada a la creatividad-y al reconocimiento que allí se obtiene de parte de aquellas/os que son clientes/as. Sin embargo, estas mismas características hacen de este tipo de inserciones una opción de difícil acceso, o al menos, una en la cual los requisitos de ingreso no resultan asequibles fácilmente.

En efecto uno de estos requerimientos es la formación profesional, que supone una inversión tanto de dinero (curso, herramientas de trabajo, etc.) como de tiempo. Por empezar, las capacitaciones en peluquería consisten en formaciones extensas e intensas (entre 3 meses a 1 año -el curso básico-, con una frecuencia semanal de hasta diez horas, o más, y luego los cursos de perfeccionamiento que pueden tener una duración similar). Esto supone que quienes las realizan puedan tener disponibilidad para planificar la organización cotidiana de sus familias. Ya que tal como hemos visto durante el trabajo de campo en la escuela de peluquería, la presencia de mujeres con hijos/as menores a cargo, es mayoritaria. En el caso de las jóvenes sin hijos/as, muchas veces deben compatibilizar sus horarios de acuerdo a las obligaciones de cuidado de hermanos/as menores. Es decir, resulta fundamental disponer de tiempo para cursar y estudiar, tiempo que se "resta" a las actividades reproductivas y que muchas veces supone conflictos dentro del hogar para estas mujeres, como detalla Cele:

Con el más chico, porque la del medio vuelve sola del colegio, me avisa cuando llega a casa. La más grande busca al nene a las cuatro. Los lunes y miércoles no hacen nada, la nena tiene coro, va por acá, me espera y vamos juntas. El nene 
tiene fútbol los días que salgo temprano. Lo llevo yo. Gracias a dios me organicé bien y puedo cumplir.

Cele es una de las alumnas de la escuela de formación en peluquería donde hicimos nuestro trabajo de campo, es madre de dos niñas de 9 y 14 años, y un varón de 4. Recientemente separada del padre de dos de sus hijos, al momento del trabajo en la escuela, ambos compartían la misma casa. Cele es trabajadora doméstica, empleo que compatibiliza con la asistencia diaria a la escuela por las tardes. Su testimonio da cuenta de lo que referimos anteriormente, ya que para cursar en la escuela, y cumplir con los horarios exigidos (cinco veces por semana, cuatro horas diarias), Cele necesita desplegar una organización doméstica que en su caso se apoya solamente en sus hijas mayores. Si bien el padre de sus hijos, habita la misma casa, no está presente en la dinámica de responsabilidades organizativas del hogar. Así, esta primera barrera de "acceso", la disponibilidad para poder completar la capacitación, en el caso de las mujeres es indesligable de las responsabilidades de cuidado 8 .

A su vez, cabe señalar que en el caso de las mujeres que consiguen realizar el curso, si bien en general esta carrera permite inserciones más o menos prontas en el mercado de trabajo, se trata de contratos precarios, en donde deben facturar por el trabajo realizado y cobran de acuerdo a un porcentaje previamente establecido. Diana, tiene 26 años, y en el momento en el que la entrevistamos trabajaba como manicura en un salón de Recoleta, un barrio de clase alta del centro de la ciudad de Buenos Aires, previamente había tenido otras breves contrataciones como manicura en peluquerías de la ciudad, y durante un tiempo más extenso en el salón de su tía.

Empecé a estudiar porque no sabía qué estudiar en la facultad, y me metí en pelu para ver qué onda. Mi abuela y la tía de mi mama eran peluqueras. Se me ocurrió porque no sabía qué hacer. Hice un curso de 4 meses, no me gustó, porque era muy comercial. Y mi mamá le dijo a la esposa de mi tío si no me quería para barrer, porque tenía una peluquería, y ahí empecé con ella. Y después terminé el curso en otra academia que es IAP [Instituto Argentino de Peluquería, academia privada de formación], como yo tenía una base estuve unos 8 meses, después hice perfeccionamiento en color. Me fui a Chile [a donde tiene familia], volví, y empecé a buscar trabajo por internet y fui a parar a la peluquería del barrio de Congreso [barrio céntrico de la ciudad].

El testimonio de Diana deja entrever una de las características fundamentales de este tipo de ocupación, la precariedad de las formas de contratación y de

8 En el caso de los varones asistentes al curso de formación, que conformaban la minoría del grupo de alumnos/ as, el tiempo se distribuía entre el trabajo rentado y la escuela. 
las condiciones de trabajo en las que es realizado. La gran mayoría se inserta en los servicios de estética y peluquería a través ya sea de un puesto no registrado, "en negro", o con contratos a tiempo parcial, facturando por servicio realizado, o como empleada/o pero con un salario básico muy bajo y el resto a porcentaje sobre trabajo realizado. Esos porcentajes fijados por el convenio colectivo, suelen variar en función del tipo de tarea que define el puesto de cada trabajador/a: colorista, peinador/estilista, asistente, manicura, pedicura, etc. Correspondiendo el porcentaje y salario más elevado al colorista, que en el caso de muchos salones, suele ser varón.

De esta manera, observamos que, a pesar de la calificación obtenida, y necesaria, la formación no garantiza el acceso a un "buen empleo", sino que por el contrario, se accede a puestos precarios, de alta rotación, jornadas extensas, lo cual redunda en pasajes de pocos meses por salones que si, por un lado ofrecen "un puesto asalariado", lo hacen con bajos sueldos, con porcentajes que se cobran "en negro", y escaso cumplimiento con los aportes y obligaciones patronales. En los relatos recabados durante el trabajo de campo, si bien se repetía esta caracterización acerca de las condiciones de empleo desfavorables, esto no redundaba necesariamente en una estigmatización de la actividad desarrollada, sino solo en el tipo de relación laboral. De esa forma, se argumentaban los cambios sucesivos de lugar de trabajo, las trayectorias por diversos salones, que son percibidos en términos de pasajes "necesarios" para la adquisición de experiencia profesional, es decir como una manera de seguir aprendiendo a trabajar. Lali, peluquera e instrumentista, de 30 años, cuenta su experiencia en el paso por estos salones:

Más de 10 horas parada... y llevabas chauchas en el día, ¿entendés? Entonces... no... no duré mucho. Hacía cortes y peinados en la peluquería... Igual aprendí un montón. Y es este chico que yo hoy en día tengo que hacerme algo en el pelo o me tengo que peinar y voy con él. Aprendí un montón de él. Cuando estás en una lugar así, es donde más aprendés... que además de un curso o de estudiar, porque... lo diario y estando ahí... es donde la práctica es lo que más te lleva a aprender cosas de peluquería, más siendo asistente. Pero no duré mucho. Habré durado 3 meses, más o menos.

A su vez, Ana, peluquera de 36 años, de la ciudad de Buenos Aires, también se refiere al aprendizaje que implica una experiencia de trabajo en un salón:

Vos lavando el pelo te das cuenta qué tipo de pelo tiene, porque... Está bien, vos hacés un curso, pero no tenés la experiencia, ¿no? Entonces... en la peluquería empezás como... se empieza abrir el abanico. Ah... y esta que tiene rulos, el pelo es más bien grueso, es más bien poroso... Eso es también, si bien uno está atento, lo vas adquiriendo de alguna manera, no sé, natural, inconsciente, ¿no? 
(...) Hasta que un día te das cuenta que decís: "Ah, no, pero vos tenés el pelo poroso..." Y decís ah, pero... es de lavar tantas cabezas... Es de sacar tanto los pelos, ¿viste? Sí, es como es más de experimentar... de darte cuenta.

Como vemos en estos testimonios, en cierta forma el aprendizaje, la posibilidad de seguir aprendiendo, justifican ocupaciones precarias e inestables, que son toleradas en tanto son percibidas como la manera de construir una trayectoria profesional en el sector, la cual se coronaria con la posibilidad de trabajar de manera independiente. Este tipo de modelo de trayecto profesional se repitió a lo largo de las diversas entrevistas realizadas. Un ejemplo de ello sean tal vez, los múltiples testimonios recabados en el marco de la escuela de peluquería. Durante las charlas mantenidas en nuestras observaciones, entre las alumnas se repetía este tipo de expectativa en relación a las carreras imaginadas para sí una vez finalizada la formación: obtener un empleo en un salón en donde, aun siendo conscientes de las malas condiciones de contratación y trabajo, podrían adquirir experiencia profesional para luego "armar el local propio":

Cele me cuenta que le cortó el pelo a un vecino. (...) Dice que de a poco se va a animar a ofrecer más. Que en el verano tiene ganas de trabajar en una peluquería, para practicar. Yo le digo: “como una pasantía” ¡Claro! ¡Para aprender!” (Registro de campo, Escuela, 20 de octubre de 2017)

Así, las valoraciones de las actividades de estética en términos de autonomía e independencia dan cuenta del importante peso que tiene en las vidas de las mujeres y en la posibilidad de construir carreras y movilidades, el manejo del tiempo propio. La flexibilidad en términos de horarios y disponibilidad, les permite acomodar el cuidado de sus hijos/as y su ocupación. Aunque no solamente, ese local propio también aparece ligado a las aspiraciones vinculadas a la posibilidad de desplegar un trabajo "creativo" y placentero, en donde dedicarle a cada clienta/e el tiempo necesario para realizar un buen trabajo y que ésta/e salga satisfecha/o. Ana, con una experiencia de más de 10 años en peluquería se refería a ello de esta forma:

(...) porque ese es el objetivo y ese es tu logro de haber... de ver el resultado... Y eso, de disfrutarlo, yo los re disfruto. Disfruto cada resultado, ¿no? Y eso del disfrute de tu clienta que se va re satisfecha, ¿no? Y cuando no va satisfecha, es un garrón, también, ¿no? (Risa leve) Pero bueno, de estar presente para poder darle... Qué sé yo, no se va satisfecha y de poder decirle: "Bueno, vení la semana que viene y vamos buscando lo que vos viniste, lo que querías"

¿Pero, si las condiciones laborales son las mismas, e incluso a veces menos estables, que aquellas que encontramos en otras ocupaciones disponibles, qué 
encierra como promesa el trabajo en los servicios de estética? Hay dos dimensiones que en nuestros estudios exploratorios aparecen como centrales en estas actividades, siempre desde la perspectiva de las trabajadoras: por un lado la idea de "ascenso" que ofrece la peluquería. Esta imagen de ascenso social no remite directamente a una mayor generación de ingresos sino a un tipo de trabajo, a una tarea vinculada a los servicios de estética y cuidado personal que vehiculizan esa idea de ascenso social a través del reconocimiento que ese trabajo posibilita, se trata de una actividad creativa, donde el producto realizado puede ser admirado, disfrutado, por otros/as. Y eso nos lleva al trabajo emocional que implica esta actividad, un trabajo emocional que se relaciona con el placer/ bienestar (de dar y recibir). Como ya ha sido señalado por Arango, entre otras autoras, gran parte del trabajo que se desarrolla en el sector de servicios de estética y peluquería consiste en un trabajo de cuidado y un trabajo emocional que versa sobre "hacer sentir bien" a el o a la cliente/a, "administrando con precaución el grado de intimidad" (Arango, 2011). Se trata de la dimensión relacional característica de estas actividades en donde el vínculo con la clienta, la escucha, la contención en muchos casos y el brindar un servicio que genera en éstas un sentimiento de placer y bienestar consigo mismas, resultan en un trabajo percibido como gratificante por quien lo realiza. Todo ello deriva en el reconocimiento y prestigio que se adquiere o es pasible de ser adquirido, a través de esta profesión:

Lo que más me conmovió (del aprendizaje) es como tenés que agarrar la tijera, estar vos concentrada y tomar la decisión de llegar al corte. Ya podemos hacer el corte que queremos pero también es flashearse con nosotras mismas. Es... no tendría las palabras justas pero es lindo (...) Tiene algo de creación, motivación todo, tenés que estar bien vos y segura para transmitir a la otra persona que se sienta segura que le vas a hacer un corte bueno que le va a quedar lindo. Siempre estar bien, concentrada, ¡porque si le demostrás a las clientas que tenés miedo a hacer un corte se te va la clienta y no tenés más! Una que se va enojada no entran diez, la que se va conforme te trae cinco o seis.

Así como Cele se refiere a la posibilidad de ser creativa presente en la peluquería, esta dimensión fue resaltada a lo largo de otras entrevistas y observaciones, confirmando el señalamiento de Arango (2011a) en su estudio sobre peluquerías en Colombia. El trabajo realizado contempla una doble dimensión que se traslada así a la manera en que es percibido y vivido, no solo por quienes lo ejecutan sino también, y en parte allí reside la diferencia con otras actividades, en la percepción social del mismo. El trabajo en los servicios de cuidados personales habilita a través de la formación, el aprendizaje de un oficio, de una profesión, que resulta valorada en el momento de la prestación, de atender a las clientas, de 
peinar, cortar, hacer el color, es decir en la puesta en práctica de una técnica específica. Pero también en el despliegue de un trabajo emocional en relación a esa clienta, para hacerla sentir bien con ella misma, y es en esa dimensión relacional en donde se juega el reconocimiento social que las mujeres que se emplean en esta actividad valoran. Todo ello, se ve reflejado en la forma en que es percibida la movilidad laboral para quienes construyen sus trayectos hacia el sector.

De esta manera, el estudio del trabajo en este sector permite observar la existencia de una movilidad percibida y proyectada como ascendente, no tanto en términos de estabilidad en el empleo y condiciones de trabajo sino en términos de prestigio, independencia y posibilidades de proyección hacia el futuro.

\section{Conclusiones}

En este artículo analizamos las inserciones de mujeres de sectores populares en el mercado de trabajo, prestando especial atención a las ocupaciones de cuidados. Desde nuestra perspectiva, estudiar los desplazamientos de las trabajadoras a través de estas ocupaciones permite observar tanto los condicionamientos que configuran las trayectorias laborales, como los márgenes de elección y los factores que intervienen en esas elecciones. Estos márgenes de elección se dan en contextos fuertemente acotados. Como vimos estas ocupaciones aparecen como el principal destino de las mujeres menos favorecidas. Estas trayectorias se configuran a través de dos elementos fundamentales, que están estrechamente ligados: las posibilidades de articulación de las actividades laborales y las responsabilidades reproductivas y el acceso a instancias de formación. En el caso de las trabajadoras que se desplazan del servicio doméstico hacia la limpieza no doméstica, estas responsabilidades reproductivas en sus propios hogares limitan el acceso a puestos con una mayor carga horaria y mayores niveles de formalidad en términos de contratación. Y a su vez, la posibilidad de acceder a experiencias de formación que como vimos en el caso de la peluquería pueden ser particularmente exigentes en términos de la dedicación horaria. Tanto en la limpieza no doméstica como en las actividades de peluquería estas instancias de formación resultan centrales. Sin embargo aparecen de dos maneras diferentes. En la limpieza no doméstica, las decisiones de formación están imbricadas con el ejercicio de los puestos en los que se desempeñan. En el caso de la peluquería, se trata de decisiones personales ligadas a un proyecto de carrera laboral.

Por otra parte, hemos visto que en ambas ocupaciones se juegan aspiraciones o expectativas diferentes en términos de las movilidades que construyen las mujeres que transitan por ellas. En el caso de la limpieza no doméstica observamos que el acceso a este tipo de puestos supone en muchos casos empleos más esta- 
bles, regulados, por fuera del espacio doméstico familiar, pero que también implican un mayor desgaste físico, procesos de trabajo sometidos a reglas más precisas y estandarizadas, que se contraponen a la flexibilidad de horarios y procesos del espacio de la limpieza doméstica. Mientras que en el caso del trabajo en los servicios de estética y peluquería, esos puestos precarios, mal pagos, con jornadas extensas, e incluso realizados "a domicilio" son acompañados de una capacitación en un oficio creativo, en donde el trabajo comprende no solo una dimensión relacional afectiva sino también la posibilidad de mostrar un producto valorado que "hace sentir bien" a el/ la cliente/a. De esta manera, en el caso de la limpieza el acceso a un puesto con mejores condiciones de empleo no es percibido como un cambio significativo en los posicionamientos de las trabajadoras en el mercado de trabajo: se percibe más como una movilidad horizontal y fronteriza entre puestos del mismo tipo. En cambio, en el caso de los servicios de estética y peluquería, a pesar de la existencia de condiciones de trabajo precarias, inestables e informales, estas inserciones son percibidas como un claro mejoramiento en su inserción. Así, las percepciones acerca de la movilidad laboral en ambos casos no aparecerían signadas, al menos no exclusivamente, por las condiciones materiales de trabajo sino que está fuertemente atravesada por la concepción subjetiva del trabajo, que en ambos casos se vincula a la manera en que éste es valorado socialmente, percepción que se construye desde determinadas posiciones de clase, género y racialidad. En definitiva, estas percepciones acerca de la movilidad están asociadas a la posibilidad de construir un espacio de reconocimiento en el trabajo.

\section{Bibliografía}

Arango Gaviria, Luz Gabriela (2011) "Género, belleza y pretensiones artísticas en el campo de las peluquerías" en Revista Latina de Sociología, 1: 1-44.

- (2011a) "Género, trabajo emocional y corporal en peluquerías y salones de belleza”, en La manzana de la discordia, Enero-Junio, (6) 1: 9-24.

Barrancos, Dora y Goren, Nora (2002) “Género y empleo en el Gran Buenos Aires. Exploraciones acerca de las calificaciones en mujeres de los sectores de pobreza", en Forni, Floreal (comp.), De la exclusión a la organización. Hacia de la integración de los pobres en los nuevos barrios del conurbano bonaerense, Buenos Aires: Ciccus.

Bertranou, Fabio y Casanova, Luis (2013), Informalidad laboral en Argentina: segmentos críticos y políticas para la formalización, Buenos Aires, OIT.

Brétin, Hélène (2001) "Le nettoyage, aux confins du jour et de la nuit". Les annales de la recherche urbain, 87: 85-99. 
Brites, Jurema (2001) "Afeto, desigualdade e rebeldia: Bastidores do serviço doméstico". Tese de Doutorado, Programa de Pós-Graduação em Antropologia Social, Porto Alegre: UFRGS.

CEPAL (2014), Panorama social de América Latina, Santiago de Chile: Naciones Unidas.

Cerrutti, Marcela y Alameijeiras Analía (2016) "La intermitencia en la participación laboral de las mujeres veinte años después: el caso de Área Metropolitana de Buenos Aires", Anais do XX Encontro Nacional de Estudos Populacionais: Iguazú.

Chaney, Elsa y García Castro, Mary (Eds) (1993) Muchacha, cachifa, criada, empleada, empregadinha, sirvienta y...más nada. Caracas: Editorial Nueva Sociedad.

Contartese, Daniel y Maceira, Verónica (2005), Diagnóstico sobre la situación laboral de las mujeres, Buenos Aires, Subsecretaría de Programación Técnica y Estudios Laborales, MTEySS.

Cortés, Rosalía (2012) "Políticas laborales y transferencias de ingresos: ¿estrategias complementarias?", Voces del Fénix, n. 5, pp: 44-51.

DÁvalos, Patricia (2013), "La Asignación Universal por Hijo desde la perspectiva de los hogares perceptores" en Estudios el Trabajo No.45, Primer Semestre, pp: 5-32.

Garazi, Débora (2014) “Trayectorias laborales, trabajo estacional e identidades: las mucamas de hotel en Mar del Plata (1960-1980)". VIII Jornadas de Sociología de la UNLP, diciembre de 2014, Ensenada, Argentina. En Memoria Académica. Disponible en: <http://www.memoria.fahce.unlp.edu.ar/ trab_eventos/ev.4440/ev.4440.pdf> (consultado: 20 de abril de 2017).

Hondagneu-Sotelo, Pierrette (2007) Domestica: Immigrant Workers Cleaning and Caring in the Shadows of Affluence. Berkeley: University of California Press.

Jaunait, Aunait y Chauvin, Sebastien (2012) Représenter l'intersection: les théories de l'intersectionnalité à l'épreuve des sciences sociales, Faculty FMG: Amsterdam Institute for Social Science Research (AISSR).

Jelin, Elizabeth (2010) Pan y afectos. La transformación de las familias, Buenos Aires, Fondo de Cultura Económica.

Messing, Karen (2016), Les souffrances invisibles. Pour une science du travail à l'écoute des gens. Montréal: Écosociété.

Micha, Ariela y Pereyra, Francisca (2017) "Las condiciones laborales de las mujeres de sectores populares en Argentina: Sobre características objetivas y 
vivencias subjetivas" en VII Jornadas de Sociología, Universidad Nacional General Sarmiento, Los Polvorines, Buenos Aires 18 y 19 de mayo.

Molinier, Pascale (2005) “Le care à l'épreuve du travail. Vulnérabilités croisées et savoir-faire discrets". P. Paperman et S. Laugier (sous la direction de) Le souci des autres. Ethique et politique du care. Paris: Editions de l'Ecole des Hautes Études en Sciences Sociales

Novick, Marta; Rojo, Sofía y Castillo, Victoria (comps.) (2008), El trabajo femenino en la post-convertibilidad. Argentina 2003-2007, Santiago de Chile: CEPAL, GTZ, Ministerio de Trabajo, Empleo y Seguridad Social.

Pereyra, Francisca (2017), Trabajadoras domésticas y protección social en la Argentina: avances $y$ desafios pendientes, Buenos Aires: OIT

PNUD (2014), Género en el trabajo. Brechas en el acceso a puestos de decisión, Aportes para el desarrollo bumano en Argentina, No 8, Buenos Aires: PNUD

Rollins, Judith (1985) Between women. Domestics and their employers. Philadelphia: Temple University Press.

Romero, Mary (2002) Maid in USA. New York and London: Routledge.

Tizziani, Ania (2011) “De la movilidad ocupacional a las condiciones de trabajo. Algunas reflexiones en torno a diferentes carreras laborales dentro del servicio doméstico en la ciudad de Buenos Aires", en Revista Trabajo y Sociedad, UNSE (XV), 17: 309-328

Tizziani, Ania y Gorban, Débora (2014) "Employers in the domestic service: between estrangement and tension”, Women's Studies International Forum, (46) 54-62. doi:10.1016/j.wsif.2014.01.001

Tronto, Joan (2015), Who Cares. How to Reshape a Democratic Politics. Londres: Cornell University Press.

Wainerman, Catalina (2002) Familia, trabajo y género. Un mundo de nuevas relaciones, Buenos Aires: UNICEF/Fondo de Cultura Económica.

Willis, Paul (1988) Aprendiendo a trabajar. Cómo los chicos de la clase obrera consiguen trabajos de clase obrera. Akal: Madrid.

\section{Otras fuentes consultadas}

Clasificador Nacional de Ocupaciones, Instituto Nacional de EsTAdísticas y Censos, Argentina: <http://www.indec.gov.ar/ftp/cuadros/ menusuperior/eph/EPHcontinua_CNO2001_reducido_09.pdf >

Encuesta Permanente de Hogares, tercer trimestre de 2016

L'Oreal Beauty Report (2016) «Belleza Por Un Futuro », gacetilla N², 22 de noviembre 